\title{
Tracking solvent dynamics during carbonic anhydrase catalysis
}

\author{
$\mathrm{C} \mathrm{Kim}^{1}$ \\ ${ }^{1}$ Ulsan National Institute of Science and Technology, Ulsan, Korea \\ cukim@unist.ac.kr
}

Human carbonic anhydrase II (CA II) is a zinc metalloenzyme that catalyzes the reversible hydration/dehydration of $\mathrm{CO} 2 / \mathrm{HCO} 3$-. Although CA II has been extensively studied to investigate the proton-transfer process that occurs in the active site, its underlying mechanism is still not fully understood. In this presentation, we present the crystallographic structures of CA II cryocooled under various $\mathrm{CO} 2$ pressures. The structures reveal new intermediate solvent states of CA II that provide crystallographic snapshots during the restoration of the protontransfer water network in the active site. In addition, the structures provide hints for the role of metal ions in CA II catalysis.

Acta Cryst. (2020). A76, a93 